\title{
Research on the Development and Utilization of Archives Information Resources Based on User's Perspective
}

\author{
Liying Cui ${ }^{1, a}$ and Chunling $\mathrm{Hu}^{2}$, b \\ 1,2 Jilin Agriculture University Changchun, China,130118 \\ acuiliying07@126.com, ${ }^{\mathrm{b}} 434228805 @ q q . c o m$
}

Keywords: User; Perspective; Archival Information Resources; Development and utilization

\begin{abstract}
Archives information resource is an important part of information resources, which contains abundant information. With the advent of the information age, the importance of the value of archives is becoming more and more popular. The development and utilization of archives information resources is highly valued. Based on the analysis of the archives information resources, this paper puts forward some suggestions for the development and utilization of the archives information for the society and the public.
\end{abstract}

\section{Introduction}

With the development of information technology, the mankind has entered the information society. Archives is an important information resource, archive information is the "original ecology", the most reliable resources, with unparalleled development and utilization value. Archives reflect the whole process of human understanding of nature and the use of nature, society and the transformation of society [1]. In the information resources, the status of archives information is irreplaceable. It is very important to promote the development and utilization of archives information resources and provide high quality archival information service for the society.

In view of the present situation of the development and utilization of archives information resources, this precious information resource has not been fully developed and utilized. Users have always been in awe of the file information, the use of archival information to maintain a certain distance attitude. Seize the opportunity, meet the challenges, with the help of new technology, new ideas, new awareness, the depth of the user to explore the file information resources, in order to improve the utilization of archival information resources, services in the community, serve the public.

\section{User Analysis in the Development and Utilization of Archives Information Resources}

Development and utilization of information archives is not only to satisfy the government, decision-making departments reference and query needs, meet the research needs, satisfy the professional service needs, to meet the public security organs, lawyers, journalists and other related professionals need to survey etc. With the advent of the information age, the use of information resources is more frequent. File resources are no longer on the shelf, the service level of private enjoyment of resources, and become accessible to everyone, the use of shared resources. Public demand for the use of archival information is also varied. In addition to daily needs, but also includes knowledge accumulation, public education, etc. Massive file information resources to meet the various needs of different audiences [2]. According to the different characteristics of the user in the process of the use of archival information, it is divided into two categories: traditional official users and emerging private users:

Traditional Official Users of Archives Information. The traditional official users of archives information mainly refer to the leaders of all levels of government organs, enterprises and institutions; Scientific research institutions; public security, lawyers, reporters and other professional investigators etc. This type of users in the use of archives information resources has distinct characteristics: First, the use of archival information has strong pertinence and purpose. In the use of the process, they clear the needs of the target, to understand the direction of the query, to be able to pay attention to the breadth of the information obtained, vertical depth mining; Second, it 
is very important to obtain the firsthand information for the original information, the authenticity and the reliability of the archives information resources. In order to ensure that information is accurate, reliable, rapid and timely access, often need to obtain information directly through the archivists; Third, the use of archival information professional. In order to achieve the purpose of serving the industry, the professional, the use of their own professional areas of archival information resources. Therefore, more attention to the industry, the field of literature, information, showing a professional trend; Fourth, pay attention to the high degree of cohesion of the file information, with a relatively long, sustained, stable demand. Whether it is decision-making leadership, scientific research or industry services, research and investigation, are not short, fragmented work. Only long-term tracking, sustained attention, in order to achieve the use of results.

Emerging Folk Users of Archival Information. The emerging private users of archives information mainly refer to the masses of the people who use the archival information for the purpose of knowledge accumulation and public education. Such a large number of users, a wide range of archives information system cannot be ignored by the huge user groups, the characteristics of the use of archival information resources: First, the user group is huge, diverse types of needs, the use of purpose is not clear. Therefore, the use of archival information presents the characteristics of decentralization, autonomy and randomization; Second, the use of archival information is for fun and leisure for the purpose. Mainly used to edify sentiment, to meet the spiritual needs. As a result, the requirements for the professional file information is not high, very few of the file information to expand the breadth, depth excavation; Third, the user mobility is very large. The use of archives information resources of the public has no clear objectives to guide, there is no professional, continuous research to do support, the lower the degree of cohesion of the file information.

\section{Difficulties in the Development and Utilization of Archives Information Resources}

Archives Information Resources Digital Integration Degree is Not High. Since entering the Internet era, the development and utilization of information resources integration has become the trend of development The development, utilization, dissemination and sharing of information resources are mostly in the form of digital resources. Through cloud computing, big data statistics, such as the use of integrated technology, with the help of the entire media transmission to the public view. Since entering the Internet era, the development and utilization of information resources integration has become the trend of development. The development, utilization, dissemination and sharing of information resources are mostly in the form of digital resources. Through cloud computing, big data statistics, such as the use of integrated technology, with the help of the entire media transmission to the public view. However, the status of archival information resources management, digital archives information resources is not high, and the process is slow, the collection structure is single, lack of coordination, lack of independent, unified, centralized management, failed to form the archives information database, to realize the system and digital management system, is not conducive to the retrieval and utilization of users, for the development and utilization of the archives information resources bring great inconvenience. [3]

The Backward Management Mode of "Focus on the Collection and Despising Use". Aspects of history, politics and economy, culture and so on, since ancient times, "Focus on the Collection and Despising Use" management mode and management concept is our file system model and mainstream ideology, deeply rooted in the hearts of archives. Strict and effective storage system and the world's leading ancient books protection, repair technology, was once proud of the capital of China's archival sector. However, with the advent of the information society, information resources with people's production and life aspects, as a part of archives information resources, also need to be reconsidered. "Focus on the collection" concept has been difficult to adapt to the development of today's society, "Focus on the use" is the new way of development of archives information resources. Due to the closed environment for a long time in China's archives system, the new situation of lack of insight, the new technology has a low sensitivity, in "Focus on the Collection and Despising Use" management concept, management mode of long in the mainstream, the concept of change is slow, passive, slow progress in digital archives information service mode, 
backward, low level of development and utilization and most have value, development and utilization of the archives information resources value is on the shelf, it is difficult to meet the needs and utilization of the archives information resources, it is difficult to achieve its economic value, social value, cultural value.

Backward and Passive Development and Utilization of Archival Information Service. At present, the development and utilization of archive information services are mostly passive door service. Users have access to the needs of their own home reading, archive information service model single, backward, passive. Archive information management department has always been difficult to lower the figure, so that the majority of users to understand the file, accept the file, the use of archives. In such an era of information explosion, the user can get to know the information through a variety of ways, and the archives information service mode is backward, operation convenience, and interactivity are poor, for their own publicity and promotion is not enough, that it is hard to think of the most original records of archive information users in the use of information in the process of making archives information of this precious resource long-term disgraceful. On the content of service, in order to ensure the authenticity and reliability of archival information, the development and utilization of archival information resources are more rigorous, serious, interesting, vivid and easy to read. How in today's fast food culture, curiosity ridden environment, find the way of development and utilization of archival information resources, created by the user's favorite, reliable and smart archives information resources lively, become the key to the development and utilization of archives information.

\section{The Development and Utilization of Archives Information Resources Based on Users}

In Order to Meet the Needs of Users, Optimize the Collection Structure, Accelerate the Integration of Archives Information. Optimize the structure of digital collection, the first to enrich, rich collection, in order to facilitate the user to better meet the needs of users, to provide information services for social archives. To expand the amount of information, based on the rich collection, according to the needs of users, there is a plan, step by step, the screening of the digital archives, to ensure the effective development of archival information, improve utilization.

Give full play to the advantages of the Internet, the integration of data resources, strengthen the joint between archives, can realize the data exchange and the formation of large capacity, distributed and shared file information database and constructing the open archives information platform, optimize the allocation of resources, enhance the user experience; To strengthen the archives and Library and information department and other relevant departments of the cooperation and sharing, the formation of one-stop service data, expand the scope of services, improve service quality, meet the information era of massive user archives information customization and demand. [4]

Optimize the File Information Service Model, Strengthen Interaction, Close the Distance with the User. In order to facilitate the public to understand and use of archives, archives management departments should be committed to various forms of archival information display in the public, close the distance between users and enhance the file information services.

Archives display and exhibition. Archives system, comprehensive, real show the contents of the archive to help users access to archive information. Due to the limitation of time and space, the number of audience is limited, but it has the most humanistic atmosphere, compared with the mass media, the network media, the impact of information explosion. This presentation is more realistic, a sense of presence, giving the user an unparalleled experience.

Archive information all media communication. The development and utilization of archival information should be supported by the development of the whole media. Cooperation with the mass media to build a platform for the use of archive information dissemination, in the vast amount of archive information, screening out the open archives information, the depth of the user interested in the archives information resources. According to the needs of customers, providing personalized service. Win-win cooperation with the mass media, the effective development, use, dissemination of the value of the file information, the construction of a full media communication platform for 
archival information. [5]

Interactive social platform of Archives. With the development of Internet technology, digital technology, smart phones, tablet PCs as the representative of the wireless digital terminal into our lives, become the Internet, access, browse the mainstream choice of information. [6] The development and utilization of archival information through various types of archives micro-blog, file blog, file public number and other social platforms, file type TV programs, exhibitions, policies, memorabilia and other archives to promote the development of the results of publicity. Strengthen the communication with the user, timely understanding of user needs, improve user stickiness, improve public confidence, attention, expand the influence and attractiveness of the archives information.

Analysis of Different User Needs, Do a Good Job in the Development and Utilization of Archival Information. The development and utilization of archives should not be generalized, different users have different information needs, it is necessary to carry out the development of archive information resources for different users, but also to help different users to a reasonable use of information resources. The library service or network services should be targeted to help users, grasp the different needs, to help them get to know the information, realize the humanized service, enhance the archives department social affinity. In addition, development and utilization of information archives should be with the power of big data, tell the user's personality, hobbies, reading tendency, identify the user's reading preferences and access direction, provide targeted information push and personalized service, to provide more diversified archives information service quality, for the user so, the development and utilization of archives information, user oriented work truly oriented society.

\section{References}

[1] W.Y. Li and Y.M Zhang. Talk about archives for the development of cultural value [J]. Archives Science Bulletin,2003(05).

[2] J. Yang. Under the background of informatization file conversion of potential users [J]. Shanghai archives, 2015(06).

[3] Y. Liu. File information resources development and utilization problem and countermeasure research $[\mathrm{J}]$. Chinese management informationization,2015(06).

[4] D.H. Shen and X.P. Li. Era of Internet + file information resources development and utilization model construction analysis [J]. Urban construction archives,2016(06).

[5] X. Liu. All media era development and utilization of archives information [J]. Cloud dream journal,2015(01).

[6] R.J. Ma and Y.Fei and L.Y. Wang. Social media service mode of archive users demand research[J]. Archives of Shanxi, 2016,(3). 